TRANSACTIONS OF THE

AMERICAN MATHEMATICAL SOCIETY

Volume 362, Number 2, February 2010, Pages 1097-1112

S 0002-9947(09)04970-8

Article electronically published on September 18, 2009

\title{
TOPOLOGICAL CLASSIFICATION OF GENERALIZED BOTT TOWERS
}

\author{
SUYOUNG CHOI, MIKIYA MASUDA, AND DONG YOUP SUH
}

\begin{abstract}
If $B$ is a toric manifold and $E$ is a Whitney sum of complex line bundles over $B$, then the projectivization $P(E)$ of $E$ is again a toric manifold. Starting with $B$ as a point and repeating this construction, we obtain a sequence of complex projective bundles which we call a generalized Bott tower. We prove that if the top manifold in the tower has the same cohomology ring as a product of complex projective spaces, then every fibration in the tower is trivial so that the top manifold is diffeomorphic to the product of complex projective spaces. This gives supporting evidence to what we call the cohomological rigidity problem for toric manifolds, "Are toric manifolds diffeomorphic (or homeomorphic) if their cohomology rings are isomorphic?" We provide two more results which support the cohomological rigidity problem.
\end{abstract}

\section{INTRODUCTION}

A toric variety $X$ of dimension $n$ is a normal complex algebraic variety with an action of an $n$-dimensional algebraic torus $\left(\mathbb{C}^{*}\right)^{n}$ having a dense orbit. A fundamental result in the theory of toric varieties says that there is a one-to-one correspondence between toric varieties and fans. It follows that the classification of toric varieties is equivalent to the classification of fans up to isomorphism.

Among toric varieties, compact smooth toric varieties, which we call toric manifolds, are well studied. Recently the second author has shown in [7] that toric manifolds as varieties can be distinguished by their equivariant cohomology. So we are led to ask how much information ordinary cohomology contains for toric manifolds and we posed the following problem in [9]. Throughout this paper, an isomorphism of cohomology rings is as graded rings unless otherwise stated.

Cohomological rigidity problem for toric manifolds. Are toric manifolds diffeomorphic (or homeomorphic) if their cohomology rings are isomorphic?

If $B$ is a toric manifold and $E$ is a Whitney sum of complex line bundles over $B$, then the projectivization $P(E)$ of $E$ is again a toric manifold. Starting with $B$ as a point and repeating this construction, say $m$ times, we obtain a sequence of

Received by the editors September 16, 2008.

2000 Mathematics Subject Classification. Primary 57R19, 57R20, 57S25, 14M25.

Key words and phrases. Generalized Bott tower, cohomological rigidity, toric manifold.

The first author was partially supported by the second stage of Brain Korea 21 project, KAIST in 2007, the second author was partially supported by Grant-in-Aid for Scientific Research 410217540092 , and the third author was partially supported by the SRC program of Korea Science and Engineering Foundation R11-2007-035-02002-0.

(C)2009 American Mathematical Society Reverts to public domain 28 years from publication 
toric manifolds:

$$
B_{m} \stackrel{\pi_{m}}{\longrightarrow} B_{m-1} \stackrel{\pi_{m-1}}{\longrightarrow} \cdots \stackrel{\pi_{2}}{\longrightarrow} B_{1} \stackrel{\pi_{1}}{\longrightarrow} B_{0}=\{\text { a point }\},
$$

where the fiber of $\pi_{i}: B_{i} \rightarrow B_{i-1}$ for $i=1, \ldots, m$ is a complex projective space $\mathbb{C} P^{n_{i}}$. We call the above sequence a generalized Bott tower of height $m$ and omit "generalized" when $n_{i}=1$ for every $i\left([4)\right.$. We also call $B_{k}$ in the tower a $k$-stage generalized Bott manifold and omit "generalized" as well when $n_{i}=1$ for every $i$.

We note that $H^{*}\left(B_{m}\right)$ is isomorphic to $H^{*}\left(\prod_{i=1}^{m} \mathbb{C} P^{n_{i}}\right)$ as a group but not necessarily as a graded ring. If every fibration in the tower (1.1) is trivial, then $B_{m}$ is diffeomorphic to $\prod_{i=1}^{m} \mathbb{C} P^{n_{i}}$ and $H^{*}\left(B_{m}\right)$ is isomorphic to $H^{*}\left(\prod_{i=1}^{m} \mathbb{C} P^{n_{i}}\right)$ as a graded ring. The following theorem shows that the converse is true and generalizes Theorem 5.1 in [8] treating Bott towers. It also gives supporting evidence to the cohomological rigidity problem above.

Theorem 1.1. If $H^{*}\left(B_{m}\right)$ is isomorphic to $H^{*}\left(\prod_{i=1}^{m} \mathbb{C} P^{n_{i}}\right)$ as rings, then every fibration in the tower (1.1) is topologically trivial; in particular, $B_{m}$ is diffeomorphic to $\prod_{i=1}^{m} \mathbb{C} P^{n_{i}}$.

Remark 1.2. It is shown in 2] that a toric manifold $X$ whose cohomology ring is isomorphic to that of a generalized Bott manifold is a generalized Bott manifold. So we can conclude that if $H^{*}(X)$ is isomorphic to $H^{*}\left(\prod_{i=1}^{m} \mathbb{C} P^{n_{i}}\right)$, then $X$ is diffeomorphic to $\prod_{i=1}^{m} \mathbb{C} P^{n_{i}}$.

2-stage Bott manifolds are famous Hirzebruch surfaces and their diffeomorphism types can also be distinguished by their cohomology rings. The following theorem generalizes this fact and gives a partial affirmative solution to the cohomological rigidity problem.

Theorem 1.3 (Corollary 6.3). 2-stage generalized Bott manifolds are diffeomorphic if and only if their cohomology rings are isomorphic.

Actually we obtain a diffeomorphism classification result for those manifolds (see Theorem 6.1) and it would be interesting to compare it with the variety classification result in [6].

We also prove the following, which gives another partial affirmative solution to the cohomological rigidity problem.

Theorem 1.4 (Theorem 7.1). 3-stage Bott manifolds are diffeomorphic if and only if their cohomology rings are isomorphic.

This paper is organized as follows. In Section 2 we recall well-known facts on projective bundles and discuss their Pontrjagin classes. We prepare two lemmas on cohomology of generalized Bott manifolds in Section 3. Section 4 is devoted to the proof of Theorem 1.1. For the proof we need to show that a Whitney sum of complex line bundles over a product of complex projective spaces is trivial if its total Chern class is trivial. This result is of independent interest and is proved in Section 5. We will discuss 2-stage generalized Bott manifolds in Section 6 and 3 -stage Bott manifolds in Section 7. In Section 8, which is an appendix, we give a sufficient condition for an isomorphism of cohomology rings with $\mathbb{Z} / 2$ coefficients to preserve Stiefel-Whitney classes. 


\section{Projective BUndLes}

Let $B$ be a smooth manifold and let $E$ be a complex vector bundle over $B$. We denote by $P(E)$ the projectivization of $E$.

Lemma 2.1. Let $B$ and $E$ be as above and let $L$ be a complex line bundle over $B$. We denote by $E^{*}$ the complex vector bundle dual to $E$. Then $P(E \otimes L), P(E)$ and $P\left(E^{*}\right)$ are isomorphic as fiber bundles over B; in particular, they are diffeomorphic.

Proof. For each $x \in B$, we choose a non-zero vector $v_{x}$ from the fiber of $L$ over $x$ and define a map $\Psi: E \rightarrow E \otimes L$ by $\Psi\left(u_{x}\right):=u_{x} \otimes v_{x}$ where $u_{x}$ is an element of the fiber of $E$ over $x$. The map $\Psi$ depends on the choice of $v_{x}$ 's but the induced map from $P(E)$ to $P(E \otimes L)$ does not because $L$ is a line bundle. It is easy to check that the induced map gives an isomorphism of $P(E)$ and $P(E \otimes L)$ as fiber bundles over $B$.

Choose a Hermitian metric $\langle$,$\rangle on E$, which is anti- $\mathbb{C}$-linear on the first entry and $\mathbb{C}$-linear on the second entry, and define a map $\Phi: E \rightarrow E^{*}$ by $\Phi(u):=\langle u$,$\rangle .$ This map is not $\mathbb{C}$-linear but anti- $\mathbb{C}$-linear, so it induces a map from $P(E)$ to $P\left(E^{*}\right)$, which gives an isomorphism as fiber bundles.

Let $y \in H^{2}(P(E))$ be minus * the first Chern class of the tautological line bundle over $P(E)$ where vectors in a line $\ell$ of $E$ form the fiber over $\ell \in P(E) . H^{*}(P(E))$ can be viewed as an algebra over $H^{*}(B)$ via $\pi^{*}: H^{*}(B) \rightarrow H^{*}(P(E))$ where $\pi: P(E) \rightarrow$ $B$ denotes the projection. When $H^{*}(B)$ is finitely generated and torsion free (this is the case when $B$ is a toric manifold), $\pi^{*}$ is injective and $H^{*}(P(E))$ as an algebra over $H^{*}(B)$ is known to be described as

$$
H^{*}(P(E))=H^{*}(B)[y] /\left(\sum_{q=0}^{n} c_{q}(E) y^{n-q}\right),
$$

where $n$ denotes the complex fiber dimension of $E$. If we formally express

$$
c(E)=\prod_{i=0}^{n}\left(1+u_{i}\right)
$$

then the relation in (2.1) is written as

$$
\sum_{q=0}^{n} c_{q}(E) y^{n-q}=\prod_{i=0}^{n}\left(y+u_{i}\right),
$$

and the total Chern class of the tangent bundle along the fibers $T_{f}(P(E))$ of $P(E)$ is given by

$$
c\left(T_{f} P(E)\right)=\sum_{q=0}^{n}(1+y)^{n-q} c_{q}(E)=\prod_{i=0}^{n}\left(1+y+u_{i}\right) ;
$$

see [1, (2) on p.515]. It follows that the total Pontrjagin class of $T_{f}(P(E))$ is given by

$$
p\left(T_{f} P(E)\right)=\prod_{i=0}^{n}\left(1+\left(y+u_{i}\right)^{2}\right) .
$$

\footnotetext{
*Our $y$ corresponds to $-\gamma_{1}$ in [1, (2) on p.515].
} 
Proposition 2.2. Let $E^{\prime} \rightarrow B^{\prime}$ be another complex vector bundle over a smooth manifold $B^{\prime}$ with the same fiber dimension as E. Suppose that $\varphi: H^{*}\left(P\left(E^{\prime}\right)\right) \rightarrow$ $H^{*}(P(E))$ is an isomorphism such that $\varphi\left(H^{*}\left(B^{\prime}\right)\right)=H^{*}(B)$. Then $\varphi\left(p\left(T_{f} P\left(E^{\prime}\right)\right)\right)$ $=p\left(T_{f} P(E)\right)$. If $\varphi$ satisfies $\varphi\left(p\left(B^{\prime}\right)\right)=p(B)$ in addition, then $\varphi\left(p\left(P\left(E^{\prime}\right)\right)\right)=$ $p(P(E))$.

Proof. Let $y^{\prime}$ be an element of $H^{2}\left(P\left(E^{\prime}\right)\right)$ defined similarly to $y$. Since $\varphi$ is an isomorphism and $\varphi\left(H^{*}\left(B^{\prime}\right)\right)=H^{*}(B)$, we have

$$
\varphi\left(y^{\prime}\right)=\epsilon y+w \quad \text { with } \epsilon= \pm 1 \text { and some } w \in H^{2}(B) .
$$

As in (2.2) we formally express $c\left(E^{\prime}\right)=\prod_{i=0}^{n}\left(1+u_{i}^{\prime}\right)$. Then we have the relation (2.3) and the formula (2.4) for $E^{\prime} \rightarrow B^{\prime}$ with prime.

Since $\varphi\left(\prod_{i=0}^{n}\left(y^{\prime}+u_{i}^{\prime}\right)\right)=\prod_{i=0}^{n}\left(\epsilon y+w+\varphi\left(u_{i}^{\prime}\right)\right)$ is zero in $H^{*}(P(E))$, we have an identity

$$
\prod_{i=0}^{n}\left(\epsilon y+w+\varphi\left(u_{i}^{\prime}\right)\right)=\epsilon^{n} \prod_{i=0}^{n}\left(y+u_{i}\right)
$$

in a polynomial ring $H^{*}(B)[y]$ in $y$ with $H^{*}(B)$ as the coefficient ring. Replace $y$ with $\sqrt{-1}+y$ and $-\sqrt{-1}+y$ in the identity above and multiply the resulting two identities at each side. Then we obtain an identity

$$
\prod_{i=0}^{n}\left(1+\left(\epsilon y+w+\varphi\left(u_{i}^{\prime}\right)\right)^{2}\right)=\prod_{i=0}^{n}\left(1+\left(y+u_{i}\right)^{2}\right)
$$

in the ring $H^{*}(B)[y]$, in particular, in $H^{*}(P(E))$. It follows from (2.4), (2.5) and (2.6) that

$$
\begin{aligned}
\varphi\left(p\left(T_{f} P\left(E^{\prime}\right)\right)\right) & =\varphi\left(\prod_{i=0}^{n}\left(1+\left(y^{\prime}+u_{i}^{\prime}\right)^{2}\right)\right) \\
& =\prod_{i=0}^{n}\left(1+\left(\epsilon y+w+\varphi\left(u_{i}^{\prime}\right)\right)^{2}\right) \\
& =\prod_{i=0}^{n}\left(1+\left(y+u_{i}\right)^{2}\right) \\
& =p\left(T_{f} P(E)\right) .
\end{aligned}
$$

This proves the first part of the proposition.

Since the tangent bundle $T P(E)$ of $P(E)$ decomposes into a Whitney sum of $\pi^{*}(T B)$ and $T_{f}(P(E))$, we obtain the latter part of the proposition.

We conclude this section with an observation on Pontrjagin classes of generalized Bott manifolds in (1.1). Since $\pi_{j}^{*}: H^{*}\left(B_{j-1}\right) \rightarrow H^{*}\left(B_{j}\right)$ is injective, we regard $H^{*}\left(B_{j-1}\right)$ as a subring of $H^{*}\left(B_{j}\right)$ for each $j$ so that we have a filtration

$$
H^{*}\left(B_{m}\right) \supset H^{*}\left(B_{m-1}\right) \supset \cdots \supset H^{*}\left(B_{1}\right) \text {. }
$$

Theorem 2.3. Let (1.1) be one generalized Bott tower and let

$$
B_{m}^{\prime} \rightarrow B_{m-1}^{\prime} \rightarrow \cdots \rightarrow B_{1}^{\prime} \rightarrow B_{0}^{\prime}=\{\text { a point }\}
$$

be another generalized Bott tower. If $\varphi: H^{*}\left(B_{m}^{\prime}\right) \rightarrow H^{*}\left(B_{m}\right)$ is an isomorphism which maps $H^{*}\left(B_{j}^{\prime}\right)$ onto $H^{*}\left(B_{j}\right)$ for each $j=1, \ldots, m$, then $\varphi\left(p\left(B_{j}^{\prime}\right)\right)=p\left(B_{j}\right)$ for any $j$. 
Proof. It follows from the assumption that the fiber dimensions of $B_{j} \rightarrow B_{j-1}$ and $B_{j}^{\prime} \rightarrow B_{j-1}^{\prime}$ must agree for each $j$. If $\varphi\left(p\left(B_{j-1}^{\prime}\right)\right)=p\left(B_{j-1}\right)$, then Proposition 2.2 implies that $\varphi\left(p\left(B_{j}^{\prime}\right)\right)=p\left(B_{j}\right)$. Therefore, the theorem follows by induction on $j$.

\section{Cohomology of generalized Bott manifolds}

Complex vector bundles involved in a generalized Bott tower (1.1) are Whitney sums of complex line bundles. Since $P(E \otimes L)$ and $P(E)$ are isomorphic as fiber bundles by Lemma 2.1 we may assume that at least one of the complex line bundles is trivial at each stage of the tower, that is,

$$
B_{i}=P\left(\underline{\mathbb{C}} \oplus \xi_{i}\right) \text { for } i=1, \ldots, m,
$$

where $\underline{\mathbb{C}}$ denotes the trivial complex line bundle and $\xi_{i}$ a Whitney sum of complex line bundles over $B_{i-1}$. We set $n_{i}=\operatorname{dim} \xi_{i}$.

Let $y_{i} \in H^{2}\left(B_{i}\right)$ denote minus the first Chern class of the tautological line bundle over $B_{i}=P\left(\underline{\mathbb{C}} \oplus \xi_{i}\right)$. We may think of $y_{i}$ as an element of $H^{2}\left(B_{k}\right)$ whenever $i \leq k$. Then the repeated use of (2.1) shows that the ring structure of $H^{*}\left(B_{m}\right)$ can be described as

$$
H^{*}\left(B_{k}\right)=\mathbb{Z}\left[y_{1}, \ldots, y_{k}\right] /\left(f_{i}\left(y_{1}, \ldots, y_{i}\right): i=1, \ldots, k\right)
$$

for $k=1, \ldots, m$, where

$$
f_{i}\left(y_{1}, \ldots, y_{i}\right)=y_{i}^{n_{i}+1}+c_{1}\left(\xi_{i}\right) y_{i}^{n_{i}}+\cdots+c_{n_{i}}\left(\xi_{i}\right) y_{i} .
$$

We prepare two lemmas to be used later.

Lemma 3.1. The set

$$
\left\{b y_{m}+w \in H^{2}\left(B_{m}\right) \mid 0 \neq b \in \mathbb{Z}, w \in H^{2}\left(B_{m-1}\right),\left(b y_{m}+w\right)^{n_{m}+1}=0\right\}
$$

lies in a one dimensional subspace of $H^{2}\left(B_{m}\right)$ if it is non-empty.

Proof. We have

$$
\begin{aligned}
& \left(b y_{m}+w\right)^{n_{m}+1}=\left(b y_{m}\right)^{n_{m}+1}+\left(n_{m}+1\right)\left(b y_{m}\right)^{n_{m}} w+\cdots \\
& =-b^{n_{m}+1} \sum_{q=1}^{n_{m}} c_{q}\left(\xi_{m}\right) y_{m}^{n_{m}+1-q}+\left(n_{m}+1\right)\left(b y_{m}\right)^{n_{m}} w+\cdots,
\end{aligned}
$$

where (3.2) is used at the second identity. If $b \neq 0$ and $\left(b y_{m}+w\right)^{n_{m}+1}=0$, then we see $b c_{1}\left(\xi_{m}\right)=\left(n_{m}+1\right) w$ by looking at the coefficients of $y_{m}^{n_{m}}$ at the identity above and hence $b$ and $w$ must be proportional, proving the lemma.

Lemma 3.2. Let $x=\sum_{j=1}^{m} b_{j} y_{j}$ be an element of $H^{*}\left(B_{m}\right)$ such that $b_{j} \neq 0$ for some $j$. Then $x^{n_{j}} \neq 0$ in $H^{*}\left(B_{m}\right)$.

Proof. Suppose $x^{n_{j}}=0$ on the contrary. Then $\left(\sum_{j=1}^{m} b_{j} y_{j}\right)^{n_{j}}$ must be in the ideal generated by the polynomials in (3.2) while a non-zero scalar multiple of $y_{j}^{n_{j}}$ appears in $\left(\sum_{j=1}^{m} b_{j} y_{j}\right)^{n_{j}}$ when we expand it because $b_{j} \neq 0$. However, it follows from (3.2) that if a non-zero scalar multiple of a power of $y_{j}$ appears in the ideal, then the exponent must be at least $n_{j}+1$, which is a contradiction. 


\section{Cohomologically product Generalized Bott manifolds}

The purpose of this section is to prove Theorem 1.1 in the Introduction.

We continue to use the notation of the previous section and from now until this section ends, we assume that $H^{*}\left(B_{m}\right)$ is isomorphic to $H^{*}\left(\prod_{i=1}^{m} \mathbb{C} P^{n_{i}}\right)$. Then, there is another set of generators $\left\{x_{1}, \ldots, x_{m}\right\}$ in $H^{2}\left(B_{m}\right)$ such that

$$
H^{*}\left(B_{m}\right)=\mathbb{Z}\left[x_{1}, \ldots, x_{m}\right] /\left(x_{1}^{n_{1}+1}, \ldots, x_{m}^{n_{m}+1}\right),
$$

and one has an expression

$$
y_{i}=\sum_{j=1}^{m} c_{i j} x_{j} \quad \text { for } i=1, \ldots, m \text { and } c_{i j} \in \mathbb{Z}
$$

and

$$
x_{i}=\sum_{j=1}^{m} d_{i j} y_{j} \quad \text { for } i=1, \ldots, m \text { and } d_{i j} \in \mathbb{Z},
$$

where both $C=\left(c_{i j}\right)$ and $D=\left(d_{i j}\right)$ are unimodular and $C=D^{-1}$.

Lemma 4.1. By an appropriate change of indices of $x_{i}$ 's with $n_{i}=n_{m}$, we may assume that $c_{m m}=d_{m m}= \pm 1$.

Proof. Case 1. The case where all the $n_{i}$ 's are the same. In this case, $x_{i}^{n_{m}+1}=0$ for any $i$. Since $x_{i}=d_{i m} y_{m}+\sum_{j \neq m} d_{i j} y_{j}$, where the sum lies in $H^{*}\left(B_{m-1}\right)$ by (3.1) and the $x_{i}$ 's are linearly independent, it follows from Lemma 3.1 that there is a unique $r$ such that $d_{r m} \neq 0$, and $d_{r m}$ is actually \pm 1 because $\operatorname{det} D= \pm 1$. Since all the $n_{i}$ 's are the same, we may assume $r=m$ if necessary by changing the indices of the $x_{i}$ 's, so $d_{m m}= \pm 1$ and $d_{i m}=0$ for $i \neq m$. This implies that $c_{m m}=d_{m m}= \pm 1$ and $c_{i m}=0$ for $i \neq m$ as well because $C=D^{-1}$.

Case 2. The general case. Let $S=\left\{N_{1}, \ldots, N_{k}\right\}$ be the set of all distinct elements of $n_{1}, \ldots, n_{m}$ such that $N_{1}>\ldots>N_{k}$. We can view $\left\{n_{1}, \ldots, n_{m}\right\}$ as a function $\mu:\{1, \ldots, m\} \rightarrow \mathbb{N}$ such that $\mu(i)=n_{i}$. Then $S$ is the image of $\mu$. Let $J_{\ell}=\mu^{-1}\left(N_{\ell}\right)$ for $\ell=1, \ldots, k$ and let $C_{J_{\ell}}$ and $D_{J_{\ell}}$ be the matrices formed from $c_{i j}$ and $d_{i j}$ with $i, j \in J_{\ell}$, respectively.

Since $x_{i}^{n_{i}+1}=0, d_{i j}$ must be 0 if $n_{i}<n_{j}$ by Lemma 3.2. This shows that $D=\left(d_{i j}\right)$ is a block upper triangular matrix

$$
\left(\begin{array}{cccc}
D_{J_{1}} & & & * \\
& D_{J_{2}} & & \\
& & \ddots & \\
0 & & & D_{J_{k}}
\end{array}\right)
$$

if $n_{1} \geq n_{2} \geq \cdots \geq n_{m}$, and in general is conjugate to the above by a permutation matrix.

Since $C=D^{-1}, C$ is also conjugate to a block upper triangular matrix

$$
\left(\begin{array}{cccc}
C_{J_{1}} & & & * \\
& C_{J_{2}} & & \\
& & \ddots & \\
0 & & & C_{J_{k}}
\end{array}\right)
$$

by a permutation matrix. Then a similar argument to Case 1 above can be applied to $C_{J_{\ell}}$ and $D_{J_{\ell}}$ (for $J_{\ell}$ containing $m$ ) instead of $C$ and $D$, and the lemma follows. 
We may further assume that $c_{m m}=d_{m m}=1$ if necessary by taking $-x_{m}$ instead of $x_{m}$, so that we may assume

$$
y_{m}=x_{m}+\sum_{j=1}^{m-1} c_{m j} x_{j} \quad \text { and } \quad x_{m}=y_{m}+\sum_{j=1}^{m-1} d_{m j} y_{j} .
$$

Lemma 4.2. If $H^{*}\left(B_{m}\right)$ is isomorphic to $H^{*}\left(\prod_{i=1}^{m} \mathbb{C} P^{n_{i}}\right)$, then $H^{*}\left(B_{m-1}\right)$ is isomorphic to $H^{*}\left(\prod_{i=1}^{m-1} \mathbb{C} P^{n_{i}}\right)$.

Proof. By (3.1), $H^{*}\left(B_{m-1}\right)$ agrees with $H^{*}\left(B_{m}\right)$ with $y_{m}=0$ plugged. On the other hand, $H^{*}\left(B_{m}\right)$ has an expression (4.1) by assumption. It follows from (4.3) that $H^{*}\left(B_{m-1}\right)$ agrees with the right hand side of (4.1) with the relation $x_{m}+$ $\sum_{j=1}^{m-1} c_{m j} x_{j}=0$ added. Therefore, we can eliminate $x_{m}$ using the added relation, so that we obtain a surjective homomorphism

$$
\mathbb{Z}\left[x_{1}, \ldots, x_{m-1}\right] /\left(x_{1}^{n_{1}+1}, \ldots, x_{m-1}^{n_{m-1}+1}\right) \rightarrow H^{*}\left(B_{m-1}\right) .
$$

But both sides above are torsion free and have the same rank, so the homomorphism above is an isomorphism, proving the lemma.

We need one more result for the proof of Theorem 1.1

Theorem 4.3. A Whitney sum of complex line bundles over a product of complex projective spaces is trivial if and only if its total Chern class is trivial.

The proof of Theorem 4.3 is rather long and of independent interest, so we shall give it in the next section and complete the proof of Theorem 1.1.

Proof of Theorem 1.1. We prove the theorem by induction on $m$. When $m=1$, the theorem is obvious. Assume the theorem is true for the $m-1$ case. Suppose $H^{*}\left(B_{m}\right) \cong H^{*}\left(\Pi_{j=1}^{m} \mathbb{C} P^{n_{j}}\right)$. Then $H^{*}\left(B_{m-1}\right) \cong H^{*}\left(\prod_{j=1}^{m-1} \mathbb{C} P^{n_{j}}\right)$ by Lemma 4.2. By the induction hypothesis, the generalized Bott tower (1.1) is trivial up to $B_{m-1}$; in particular, $B_{m-1}$ is diffeomorphic to $\prod_{j=1}^{m-1} \mathbb{C} P^{n_{j}}$.

Remember that $B_{m}=P\left(\underline{\mathbb{C}} \oplus \xi_{m}\right)$ and express $\xi_{m}=\bigoplus_{i=1}^{n_{m}} \eta_{i}$ where $\eta_{i}$ is a complex line bundle over $B_{m-1}$. Let $\gamma_{j}$ be the complex line bundle over $B_{m-1}$ whose first Chern class is $y_{j}$. One can write

$$
c_{1}\left(\eta_{i}\right)=\sum_{j=1}^{m-1} a_{i j} y_{j} \quad \text { with } a_{i j} \in \mathbb{Z}
$$

Then $\eta_{i}=\bigotimes_{j=1}^{m-1} \gamma_{j}^{a_{i j}}$.

The fibration $B_{m}=P\left(\underline{\mathbb{C}} \oplus \xi_{m}\right) \rightarrow B_{m-1}$ is isomorphic to a fibration $P\left(\left(\underline{\mathbb{C}} \oplus \xi_{m}\right) \otimes L\right) \rightarrow B_{m-1}$ for any complex line bundle $L$ over $B_{m-1}$. Therefore, it suffices to find a complex line bundle $L$ such that the total Chern class of $\left(\underline{\mathbb{C}} \oplus \xi_{m}\right) \otimes L$ is trivial because the triviality of the bundle follows from the triviality of the Chern class by Theorem 4.3 ,

We take $L=\bigotimes_{j=1}^{m-1} \gamma_{j}^{-d_{m j}}$ with $d_{m j}$ in (4.3). Then

$$
c\left(\left(\underline{\mathbb{C}} \oplus \xi_{m}\right) \otimes L\right)=\prod_{i=0}^{n_{m}}\left(1+\sum_{j=1}^{m-1}\left(a_{i j}-d_{m j}\right) y_{j}\right) \quad \text { in } H^{*}\left(B_{m-1}\right),
$$


where $a_{0 j}=0$. On the other hand, it follows from (3.1) and (4.4) that

$$
\begin{aligned}
f_{m}\left(y_{1}, \ldots, y_{m}\right) & =y_{m}^{n_{m}+1}+c_{1}\left(\xi_{m}\right) y_{m}^{n_{m}}+\cdots+c_{n_{m}}\left(\xi_{m}\right) y_{m} \\
& =y_{m} \prod_{i=1}^{n_{m}}\left(y_{m}+c_{1}\left(\eta_{i}\right)\right) \\
& =\prod_{i=0}^{n_{m}}\left(y_{m}+\sum_{j=1}^{m-1} a_{i j} y_{j}\right) \\
& =0
\end{aligned}
$$

in $H^{*}\left(B_{m}\right)$. We plug $y_{m}=x_{m}-\sum_{j=1}^{m-1} d_{m j} y_{j}$ from (4.3) into (4.6) to get

$$
0=\prod_{i=0}^{n_{m}}\left(x_{m}+\sum_{j=1}^{m-1}\left(a_{i j}-d_{m j}\right) y_{j}\right)
$$

in $H^{*}\left(B_{m}\right)$. Here we note that

$$
H^{*}\left(B_{m}\right)=\mathbb{Z}\left[y_{1}, \ldots, y_{m-1}, x_{m}\right] /\left(f_{1}\left(y_{1}\right), \ldots, f_{m-1}\left(y_{1}, \ldots, y_{m-1}\right), x_{m}^{n_{m}+1}\right)
$$

because a natural homomorphism from the right hand side above to $H^{*}\left(B_{m}\right)$ is surjective by (3.1) and (4.3), and hence isomorphic since both are torsion free and have the same rank. Therefore, when we expand the right hand side of (4.7), the coefficient of $x_{m}^{k}$ must be zero for any $k=1, \ldots, n_{m}$. This implies that the right hand side of (4.5) is equal to 1 , proving the theorem.

Combining Theorem 1.1 with Theorem 8.1 in 3, we obtain the following corollary, which generalizes Theorem 5.1 in [8] treating the case where $n_{i}=1$ for any $i$.

Corollary 4.4. If the cohomology ring of a quasitoric manifold over a product of simplices is isomorphic to that of $\prod_{i=1}^{m} \mathbb{C} P^{n_{i}}$, then it is homeomorphic to $\prod_{i=1}^{m} \mathbb{C} P^{n_{i}}$.

Remark 4.5. Similarly to Remark 1.2 the assumption "over a product of simplices" in the corollary above can be dropped by a result in [2].

\section{Proof of Theorem 4.3}

This section is devoted to the proof of Theorem 4.3. We recall a general fact. A more refined result can be found in [11].

Lemma 5.1. Let $X$ be a finite $C W$-complex such that $H^{\text {odd }}(X)=0$ and $H^{*}(X)$ has no torsion. Then complex $n$-dimensional vector bundles over $X$ with $2 n \geq \operatorname{dim} X$ are isomorphic if and only if their total Chern classes are the same.

Proof. The assumption on $H^{*}(X)$ implies that $K(X)$ is torsion free, so the Chern character gives a monomorphism from $K(X)$ to $H^{*}(X ; \mathbb{Q})$. On the other hand, if $\operatorname{dim} X \leq 2 n$, then the homotopy set $[X, B U(n)]$, where $B U(n)$ denotes the classifying space of a unitary group $U(n)$, agrees with $K(X)$. This implies the lemma.

Let $B=\prod_{j=1}^{k} \mathbb{C} P^{n_{j}}$ be a product of complex projective spaces and let $E=$ $\bigoplus_{i=1}^{n} \eta_{i}$ be a Whitney sum of complex line bundles $\eta_{i}$ over $B$. Suppose that $c(E)=$ 1. Then since $H^{\text {odd }}(B)=0$ and $H^{*}(B)$ has no torsion, $E$ is trivial by Lemma 5.1 when $n \geq \sum_{j=1}^{k} n_{j}$. So we assume that $n<\sum_{j=1}^{k} n_{j}$ in the following. 
By assumption

$$
H^{*}(B)=\mathbb{Z}\left[x_{1}, \ldots, x_{k}\right] /\left(x_{1}^{n_{1}+1}, \ldots, x_{k}^{n_{k}+1}\right),
$$

where we can take $x_{j}$ as the first Chern class of the pullback $\gamma_{j}$ of the tautological line bundle over $\mathbb{C} P^{n_{j}}$ via the projection $\prod_{j=1}^{k} \mathbb{C} P^{n_{j}} \rightarrow \mathbb{C} P^{n_{j}}$. Then we may assume that $\eta_{i}=\bigotimes_{j=1}^{k} \gamma_{j}^{a_{i j}}$ with $a_{i j} \in \mathbb{Z}$ and

$$
1=c(E)=\prod_{i=1}^{n}\left(1+\sum_{j=1}^{k} a_{i j} x_{j}\right)
$$

It follows that

$$
0=c_{1}(E)=\sum_{i=1}^{n}\left(\sum_{j=1}^{k} a_{i j} x_{j}\right)=\sum_{j=1}^{k}\left(\sum_{i=1}^{n} a_{i j}\right) x_{j} .
$$

Since the $x_{j}$ 's are linearly independent, the identity above implies that

$$
\sum_{i=1}^{n} a_{i j}=0 \quad \text { for each } j=1, \ldots, k .
$$

Moreover it follows from (5.1) that

$$
0=c_{2}(E)=\sum_{i^{\prime}>i=1}^{n}\left[\left(\sum_{j=1}^{k} a_{i j} x_{j}\right)\left(\sum_{j=1}^{k} a_{i^{\prime} j} x_{j}\right)\right] .
$$

We need to consider two cases.

Case I. $n_{j} \geq 2$ for some $j=1, \ldots, k$. Since $x_{j}^{2} \neq 0$ in $H^{*}(B)$ in this case, the coefficient of the $x_{j}^{2}$-term in (5.3) must vanish. Thus $\sum_{i^{\prime}>i=1}^{n} a_{i j} a_{i^{\prime} j}=0$. Therefore from (5.2) we have

$$
0=\left(\sum_{i=1}^{n} a_{i j}\right)^{2}=\sum_{i=1}^{n} a_{i j}^{2}+2 \sum_{i^{\prime}>i=1}^{n} a_{i j} a_{i^{\prime} j} .
$$

Hence $\sum_{i=1}^{n} a_{i j}^{2}=0$, which implies that

$$
a_{1 j}=\cdots=a_{n j}=0 .
$$

Case II. $n_{j}=1$ for all $j=1, \ldots, k$. In this case, $n<k$ as $n<\sum_{j=1}^{k} n_{j}$. Set $\mathbf{v}_{j}=$ $\left(a_{1 j}, \ldots, a_{n j}\right) \in \mathbb{Z}^{n}$ for $j=1, \ldots, k$. We claim that $\mathbf{v}_{j}=\mathbf{0}$ for some $j=1, \ldots, k$. Since $x_{j} x_{j^{\prime}} \neq 0$ in $H^{*}(B)$ for $j \neq j^{\prime}$, the coefficient of the $x_{j} x_{j^{\prime}}$-term in (5.3) must vanish. Namely,

$$
\begin{aligned}
0 & =\sum_{i=1}^{n} a_{i j}\left(\sum_{i^{\prime}=1}^{n} a_{i^{\prime} j^{\prime}}-a_{i j^{\prime}}\right) \\
& =\left(\sum_{i=1}^{n} a_{i j}\right)\left(\sum_{i^{\prime}=1}^{n} a_{i^{\prime} j^{\prime}}\right)-\sum_{i=1}^{n} a_{i j} a_{i j^{\prime}} .
\end{aligned}
$$

By (5.2) we have

$$
\sum_{i=1}^{n} a_{i j} a_{i j^{\prime}}=0 \quad \text { for all } 1 \leq j, j^{\prime} \leq k, \quad j \neq j^{\prime}
$$


This means that $k$ vectors $\mathbf{v}_{1}, \ldots, \mathbf{v}_{k}$ in $\mathbb{Z}^{n} \subset \mathbb{R}^{n}$ are mutually orthogonal. But since $k>n, \mathbf{v}_{j}=\mathbf{0}$ for some $j=1, \ldots, k$.

We have shown that in either case there exists some $j$ such that $\left(a_{1 j}, \ldots, a_{n j}\right)=$ 0. For simplicity, assume $j=k$. Then $\eta_{i}$ is of the form $\bigotimes_{j=1}^{k-1} \gamma_{j}^{a_{i j}}$. Let $\bar{\gamma}_{j}$ be the pull-back bundle of the tautological line bundle of $\mathbb{C} P^{n_{j}}$ via the projection $\prod_{j=1}^{k-1} \mathbb{C} P^{n_{j}} \rightarrow \mathbb{C} P^{n_{j}}$. Then $E=\left(\bigoplus_{i=1}^{n} \bigotimes_{j=1}^{k-1} \bar{\gamma}_{j}^{a_{i j}}\right) \times \mathbb{C} P^{n_{k}}$. Hence the problem reduces to the bundle on $\prod_{j=1}^{k-1} \mathbb{C} P^{n_{j}}$.

The argument above shows that the proof of the theorem reduces to the case $k=1$, so the theorem follows from the following lemma.

Lemma 5.2. Let $E$ and $E^{\prime}$ be Whitney sums of complex line bundles over $\mathbb{C} P^{n}$ of the same dimension. If $c(E)=c\left(E^{\prime}\right)$, then $E$ and $E^{\prime}$ are isomorphic.

Proof. Let $\gamma^{u}$ denote a complex line bundle over $\mathbb{C} P^{n}$ whose first Chern class is $u \in H^{2}\left(\mathbb{C} P^{n}\right)$. Then $E=\bigoplus_{i=0}^{m} \gamma^{u_{i}}$ and $E^{\prime}=\bigoplus_{i=0}^{m} \gamma^{u_{i}^{\prime}}$ with $u_{i}, u_{i}^{\prime} \in H^{2}\left(\mathbb{C} P^{n}\right)$. In case $m \geq n$, the lemma follows from Lemma 5.1. In case $m<n, c(E)=c\left(E^{\prime}\right)$ implies that $\left\{u_{0}, \ldots, u_{m}\right\}=\left\{u_{0}^{\prime}, \ldots, u_{m}^{\prime}\right\}$ and hence $E$ and $E^{\prime}$ are isomorphic.

\section{2-STAGE GENERALIZED BotT MANIFOLDS}

A 2-stage Bott manifold is a Hirzebruch surface $H_{a}=P\left(\underline{\mathbb{C}} \oplus \gamma^{a}\right)$, where $a \in \mathbb{Z}$ and $\gamma$ denotes the tautological line bundle over $\mathbb{C} P^{1}$, and it is well known that $H_{a}$ and $H_{b}$ are isomorphic as varieties if and only if $|a|=|b|$ and diffeomorphic if and only if $a \equiv b(\bmod 2)$.

2-stage generalized Bott manifolds can be thought of as a higher dimensional generalization of Hirzebruch surfaces, and their classification as varieties is completed in [6]. In this section we complete the diffeomorphism classification of those manifolds

Let $B_{1}=\mathbb{C} P^{n_{1}}$ and

$$
B_{2}=P\left(\bigoplus_{i=0}^{n_{2}} \gamma^{u_{i}}\right)
$$

where $u_{0}=0$ and $\gamma^{u_{i}}$ denotes the complex line bundle over $B_{1}$ whose first Chern class is $u_{i} \in H^{2}\left(B_{1}\right)$ as before. Similarly let

$$
B_{2}^{\prime}=P\left(\bigoplus_{i=0}^{n_{2}} \gamma^{u_{i}^{\prime}}\right)
$$

be another 2-stage generalized Bott manifold with $B_{1}=\mathbb{C} P^{n_{1}}$ as 1-stage, where $u_{0}^{\prime}=0$.

Theorem 6.1. Let $B_{2}$ and $B_{2}^{\prime}$ be as above. Then the following are equivalent.

(1) There exist $\epsilon= \pm 1$ and $w \in H^{2}\left(B_{1}\right)$ such that

$$
\prod_{i=0}^{n_{2}}\left(1+\epsilon\left(u_{i}^{\prime}+w\right)\right)=\prod_{i=0}^{n_{2}}\left(1+u_{i}\right) \quad \text { in } H^{*}\left(B_{1}\right) .
$$

(2) $B_{2}$ and $B_{2}^{\prime}$ are diffeomorphic.

(3) $H^{*}\left(B_{2}\right)$ and $H^{*}\left(B_{2}^{\prime}\right)$ are isomorphic. 
Proof. Condition (1) means that $\left(\bigoplus_{i=0}^{n_{2}} \gamma^{u_{i}^{\prime}}\right) \otimes \gamma^{w}$ or its dual has the same total Chern class as $\bigoplus_{i=0}^{n_{2}} \gamma^{u_{i}}$, so that they are isomorphic as vector bundles by Lemma 5.2. This together with Lemma 2.1 implies (2). The implication $(2) \Rightarrow(3)$ is obvious, so it suffices to prove the implication $(3) \Rightarrow(1)$.

Suppose $H^{*}\left(B_{2}\right)$ and $H^{*}\left(B_{2}^{\prime}\right)$ are isomorphic. Then there is an isomorphism

$$
\begin{aligned}
\varphi: H^{*}\left(B_{2}^{\prime}\right)=\mathbb{Z}\left[x, y^{\prime}\right] /\left(x^{n_{1}+1}, \prod_{i=0}^{n_{2}}\left(y^{\prime}+u_{i}^{\prime}\right)\right) \\
\rightarrow H^{*}\left(B_{2}\right)=\mathbb{Z}[x, y] /\left(x^{n_{1}+1}, \prod_{i=0}^{n_{2}}\left(y+u_{i}\right)\right) .
\end{aligned}
$$

Express

$$
\varphi(x)=p x+q y \quad \text { and } \quad \varphi\left(y^{\prime}\right)=r x+s y
$$

with $p, q, r, s \in \mathbb{Z}$. Since $\varphi$ is an isomorphism, we have

$$
p s-q r= \pm 1 \text {. }
$$

We distinguish three cases.

Case 1 . The case where $n_{1} \geq 2$ and $n_{2}=1$. We write $u_{1}=a x$ and $u_{1}^{\prime}=a^{\prime} x$. Since $\varphi\left(y^{\prime}\left(y^{\prime}+a^{\prime} x\right)\right)=0$ and $y(y+a x)=0$ in $H^{*}\left(B_{2}\right)$, we have

$$
\begin{aligned}
0 & =(r x+s y)\left((r x+s y)+a^{\prime}(p x+q y)\right) \\
& =r\left(r+a^{\prime} p\right) x^{2}+\left(r\left(s+a^{\prime} q\right)+s\left(r+a^{\prime} p\right)\right) x y+s\left(s+a^{\prime} q\right) y^{2} \\
& =r\left(r+a^{\prime} p\right) x^{2}+\left(r\left(s+a^{\prime} q\right)+s\left(r+a^{\prime} p\right)-s\left(s+a^{\prime} q\right) a\right) x y .
\end{aligned}
$$

Therefore,

$$
r\left(s+a^{\prime} q\right)+s\left(r+a^{\prime} p\right)=s\left(s+a^{\prime} q\right) a
$$

and moreover since $n_{1} \geq 2$, we have $r\left(r+a^{\prime} p\right)=0$ and hence $r=0$ or $r=-a^{\prime} p$.

If $r=0$, then $p= \pm 1$ and $s= \pm 1$ from (6.2) and hence $\pm a^{\prime}=\left(s+a^{\prime} q\right) a$ from (6.3), which implies that $a \mid a^{\prime}$. If $r=-a^{\prime} p$, then from (6.2) we have $\pm 1=p s-q r=$ $p s+a^{\prime} p q=p\left(s+a^{\prime} q\right)$. Thus $p= \pm 1$ and $s+a^{\prime} q= \pm 1$. From (6.3) we have $\pm a^{\prime}=s a$ and hence $a \mid a^{\prime}$. In any case we have shown that $a^{\prime}$ is divisible by $a$. By the symmetry, $a$ is divisible by $a^{\prime}$. Thus $a= \pm a^{\prime}$, and hence the identity in (1) is satisfied with $w=0$.

Case 2. The case where $n_{1}=n_{2}=1$. We write $u_{1}=a x$ and $u_{1}^{\prime}=a^{\prime} x$ as in Case 1 above. The identity in (1) is equivalent to

$$
a \equiv a^{\prime} \bmod 2 .
$$

In the following all congruence relations are taken modulo 2 unless stated otherwise. It follows from (6.3) and (6.2) that

$$
a^{\prime} \equiv s\left(s+a^{\prime} q\right) a .
$$

On the other hand, since $x^{2}=0$, the identity $\varphi(x)^{2}=0$ implies that

$$
0=(p x+q y)^{2} \equiv q^{2} y^{2} \equiv q^{2} a x y,
$$

so that

$$
q^{2} a \equiv 0 .
$$

If $a \equiv 0$, then so is $a^{\prime}$ from (6.4). If $a \equiv 1$, then $q \equiv 0$ from (6.5), so that $a^{\prime} \equiv s^{2} a$ from (6.4) and $p s \equiv 1$ (and hence $s \equiv 1$ ) from (6.2). Therefore, $a \equiv a^{\prime}$ in any case. 
Case 3. The case where $n_{2} \geq 2$. If $u_{i}=u_{i}^{\prime}=0$ for all $i$ 's, then the identity in (1) holds with $w=0$, so we may assume either $u_{i}$ or $u_{i}^{\prime}$ is non-zero for some $i$ and moreover $u_{i} \neq 0$ for some $i$ without loss of generality. Then, since $0=\varphi\left(x^{n_{1}+1}\right)=$ $(p x+q y)^{n_{1}+1}, q=0$ by Lemma 6.2 below. This means that $\varphi$ preserves the subring $H^{*}\left(B_{1}\right)$, so that we have

$$
\varphi\left(y^{\prime}\right)=\epsilon y+w \quad \text { for some } w \in H^{2}\left(B_{1}\right),
$$

where $\epsilon= \pm 1$. Therefore $\varphi\left(\prod_{i=0}^{n_{2}}\left(y^{\prime}+u_{i}^{\prime}\right)\right)=\prod_{i=0}^{n_{2}}\left(\epsilon y+w+\varphi\left(u_{i}^{\prime}\right)\right)$. Since this element vanishes in $H^{*}\left(B_{2}\right)$ and is a polynomial of degree $n_{2}+1$ in $y$, we have an identity

$$
\prod_{i=0}^{n_{2}}\left(y+\epsilon\left(w+\varphi\left(u_{i}^{\prime}\right)\right)\right)=\prod_{i=0}^{n_{2}}\left(y+u_{i}\right)
$$

as polynomials in $y$. Then, plugging $y=1$, we obtain the identity in (1) in the theorem.

Here is the lemma used above. We shall use the same notation as above.

Lemma 6.2. Assume that $n_{2} \geq 2, u_{0}=0$ and $u_{i} \neq 0$ for some $1 \leq i \leq n_{2}$. If $(\alpha x+\beta y)^{n_{1}+1}=0$ in the ring $\mathbb{Z}[x, y] /\left(x^{n_{1}+1}, \prod_{i=0}^{n_{2}}\left(y+u_{i}\right)\right)$ for some integers $\alpha, \beta$, then $\beta=0$.

Proof. Since $(\alpha x+\beta y)^{n_{1}+1}=0$ in the ring $\mathbb{Z}[x, y] /\left(x^{n_{1}+1}, \prod_{i=0}^{n_{2}}\left(y+u_{i}\right)\right)$, there are a homogeneous polynomial $g(x, y)$ in $x, y$ of total degree $n_{1}-n_{2}$ and an integer $c$ such that

$$
(\alpha x+\beta y)^{n_{1}+1}-c x^{n_{1}+1}=g(x, y) \prod_{i=0}^{n_{2}}\left(y+u_{i}\right)
$$

as polynomials in $x$ and $y$. In fact, $c=\alpha^{n_{1}+1}$ as $u_{0}=0$.

Suppose $g(x, y) \neq 0$ (so that $n_{1} \geq n_{2}$ ). When we split the left hand side into a product of linear polynomials in $x$ and $y$, it has at most two linear polynomials over $\mathbb{Z}$ as factors while the right hand side has at least three linear polynomials over $\mathbb{Z}$ as $n_{2} \geq 2$ by assumption. This is a contradiction. Therefore $g(x, y)=0$. But then $\beta$ must be zero, proving the lemma.

Corollary 6.3. 2-stage generalized Bott manifolds are diffeomorphic if and only if their cohomology rings are isomorphic.

Proof. Let $B_{2} \rightarrow B_{1}=\mathbb{C} P^{n_{1}}$ be a generalized Bott tower of height 2 where the fiber is $\mathbb{C} P^{n_{2}}$, and let $B_{2}^{\prime} \rightarrow B_{1}^{\prime}=\mathbb{C} P^{n_{1}^{\prime}}$ be another generalized Bott tower of height 2 where the fiber is $\mathbb{C} P^{n_{2}^{\prime}}$. Suppose that $H^{*}\left(B_{2}\right)$ is isomorphic to $H^{*}\left(B_{2}^{\prime}\right)$. Then $\left\{n_{1}, n_{2}\right\}=\left\{n_{1}^{\prime}, n_{2}^{\prime}\right\}$, which we can see from their Betti numbers. If $n_{i}=n_{i}^{\prime}$ for $i=1,2$, then the corollary follows from Theorem 6.1. Therefore, we may assume that $n_{1}=n_{2}^{\prime}, n_{2}=n_{1}^{\prime}$ and they are different. If both $B_{2}$ and $B_{2}^{\prime}$ are cohomological products, then they are diffeomorphic to $\mathbb{C} P^{n_{1}} \times \mathbb{C} P^{n_{2}}$ by Theorem 1.1 .

In the sequel it suffices to prove that $H^{*}\left(B_{2}\right)$ and $H^{*}\left(B_{2}^{\prime}\right)$ are not isomorphic when they are not cohomological products and $n_{1}=n_{2}^{\prime} \neq n_{2}=n_{1}^{\prime}$. We may assume $n_{1}>n_{2}$ without loss of generality. Since $B_{2}^{\prime}$ is a $\mathbb{C} P^{n_{1}}$-bundle over $\mathbb{C} P^{n_{2}}$, there is a non-zero element in $H^{2}\left(B_{2}^{\prime}\right)$ whose $n_{1}$-th power vanishes; in fact, a non-zero element in $H^{2}\left(B_{2}^{\prime}\right)$ coming from the base space $\mathbb{C} P^{n_{2}}$ is such an element because 
$n_{1}>n_{2}$. On the other hand, it is not difficult to see that there is no such non-zero element in $H^{2}\left(B_{2}\right)$ since

$$
H^{*}\left(B_{2}\right)=\mathbb{Z}[x, y] /\left(x^{n_{1}+1}, \prod_{i=0}^{n_{2}}\left(y+u_{i}\right)\right),
$$

where $u_{0}=0$ and $u_{i} \neq 0$ for some $1 \leq i \leq n_{2}$. (It also follows from Lemma 6.2 when $n_{2} \geq 2$.)

\section{3-STAGE BotT MANifOLDS}

This section is devoted to the proof of Theorem 1.4 in the Introduction, that is,

Theorem 7.1. 3-stage Bott manifolds are diffeomorphic if and only if their cohomology rings are isomorphic.

Remember a Bott tower of height 3:

$$
B_{3} \stackrel{\pi_{3}}{\longrightarrow} B_{2} \stackrel{\pi_{2}}{\longrightarrow} B_{1} \stackrel{\pi_{1}}{\longrightarrow} B_{0}=\{\text { a point }\}
$$

where $B_{i}=P\left(\underline{\mathbb{C}} \oplus \xi_{i}\right)$ for $i=1,2,3$ and $\xi_{i}$ is a complex line bundle over $B_{i-1}$. Let $\gamma_{i}$ be the dual of the tautological line bundle over $B_{i}$ and let $y_{i}$ be the first Chern class of $\gamma_{i}$. Then

$$
\xi_{1}=\underline{\mathbb{C}}, \quad \xi_{2}=\gamma_{1}^{a}, \quad \xi_{3}=\pi_{2}^{*}\left(\gamma_{1}\right)^{b} \otimes \gamma_{2}^{c}
$$

with integers $a, b, c$, and it follows from (2.1) that

$$
H^{*}\left(B_{3}\right)=\mathbb{Z}\left[y_{1}, y_{2}, y_{3}\right] /\left(y_{1}^{2}, y_{2}\left(a y_{1}+y_{2}\right), y_{3}\left(b y_{1}+c y_{2}+y_{3}\right)\right),
$$

where $y_{1}$ and $y_{2}$ are regarded as elements of $H^{*}\left(B_{3}\right)$ as before.

We note that $H^{*}\left(B_{2} ; \mathbb{Q}\right)$, which is a subring of $H^{*}\left(B_{3} ; \mathbb{Q}\right)$ with $y_{3}=0$ in (7.1), is isomorphic to $H^{*}\left(\left(\mathbb{C} P^{1}\right)^{2} ; \mathbb{Q}\right)$ because $y_{1}^{2}=0$ and $\left(a y_{1}+2 y_{2}\right)^{2}=0$. However $H^{*}\left(B_{3} ; \mathbb{Q}\right)$ is not necessarily isomorphic to $H^{*}\left(\left(\mathbb{C} P^{1}\right)^{3} ; \mathbb{Q}\right)$ as is shown in the following lemma.

Lemma 7.2. The following are equivalent.

(1) $H^{*}\left(B_{3} ; \mathbb{Q}\right) \cong H^{*}\left(\left(\mathbb{C} P^{1}\right)^{3} ; \mathbb{Q}\right)$.

(2) $\left(\sum_{i=1}^{3} a_{i} y_{i}\right)^{2}=0$ in $H^{*}\left(B_{3} ; \mathbb{Q}\right)$ for some integers (or rational numbers) $a_{i}$ with $a_{3} \neq 0$.

(3) $c(2 b-a c)=0$.

Proof. $(1) \Leftrightarrow(2)$. This equivalence follows from the observation made in the paragraph just before the lemma.

$(2) \Rightarrow(3)$. Since $y_{2}^{2}=-a y_{1} y_{2}$ and $y_{3}^{2}=-b y_{1} y_{3}-c y_{2} y_{3}$ by (7.1), we have

$$
\begin{aligned}
& \left(\sum_{i=1}^{3} a_{i} y_{i}\right)^{2}=\sum_{i=1}^{3} a_{i}^{2} y_{i}^{2}+2 \sum_{i<j} a_{i} a_{j} y_{i} y_{j} \\
& \quad=\left(2 a_{1} a_{2}-a_{2}^{2} a\right) y_{1} y_{2}+\left(2 a_{2} a_{3}-a_{3}^{2} c\right) y_{2} y_{3}+\left(2 a_{3} a_{1}-a_{3}^{2} b\right) y_{3} y_{1},
\end{aligned}
$$

so that

$$
2 a_{1} a_{2}=a_{2}^{2} a, \quad 2 a_{2} a_{3}=a_{3}^{2} c, \quad 2 a_{3} a_{1}=a_{3}^{2} b .
$$

An elementary computation shows that these imply $c(2 b-a c)=0$. 
$(3) \Rightarrow(2)$. If $c(2 b-a c)=0$, then an elementary computation shows that $\left(b y_{1}+c y_{2}+2 y_{3}\right)^{2}=0$.

Lemma 7.3. The first Pontrjagin class of $B_{3}$ is given by

$$
p_{1}\left(B_{3}\right)=c(2 b-a c) y_{1} y_{2} .
$$

Therefore, $p_{1}\left(B_{3}\right)=0$ if and only if $H^{*}\left(B_{3} ; \mathbb{Q}\right) \cong H^{*}\left(\left(\mathbb{C} P^{1}\right)^{3} ; \mathbb{Q}\right)$.

Proof. Since $p\left(B_{1}\right)=1$, it follows from (2.4) that

$$
p\left(B_{2}\right)=\left(1+y_{2}^{2}\right)\left(1+\left(y_{2}+a y_{1}\right)^{2}\right),
$$

which is equal to 1 because $y_{2}^{2}=-a y_{1} y_{2}$. Therefore, it follows from (2.4) again that

$$
\begin{aligned}
p\left(B_{3}\right) & =\left(1+y_{3}^{2}\right)\left(1+\left(y_{3}+b y_{1}+c y_{2}\right)^{2}\right) \\
& =1+y_{3}^{2}+\left(y_{3}+b y_{1}+c y_{2}\right)^{2} \\
& =1+c(2 b-a c) y_{1} y_{2},
\end{aligned}
$$

where we used $y_{3}\left(y_{3}+b y_{1}+c y_{2}\right)=0$ and $y_{2}^{2}=-a y_{1} y_{2}$. This proves the first part of the lemma. The latter part follows from the first part and Lemma 7.2

We shall complete the proof of Theorem [7.1. Let $B_{3}^{\prime} \rightarrow B_{2}^{\prime} \rightarrow B_{1}^{\prime}$ be another Bott tower of height 3 and denote the elements corresponding to $a, b, c$ and $y_{i}$ by $a^{\prime}, b^{\prime}, c^{\prime}$ and $y_{i}^{\prime}$. The results in 12 and 5 tell us that if there is an isomorphism $\varphi: H^{*}\left(B_{3}^{\prime}\right) \rightarrow H^{*}\left(B_{3}\right)$ such that $\varphi\left(p_{1}\left(B_{3}^{\prime}\right)\right)=p_{1}\left(B_{3}\right)$ and $\varphi\left(w_{2}\left(B_{3}^{\prime}\right)\right)=w_{2}\left(B_{3}\right)$, then $B_{3}$ and $B_{3}^{\prime}$ are diffeomorphic, where $w_{2}$ denotes the second Stiefel-Whitney class.

Suppose $H^{*}\left(B_{3}^{\prime}\right) \cong H^{*}\left(B_{3}\right)$ and let $\varphi: H^{*}\left(B_{3}^{\prime}\right) \rightarrow H^{*}\left(B_{3}\right)$ be an isomorphism. Since $\varphi\left(w_{2}\left(B_{3}^{\prime}\right)\right)=w_{2}\left(B_{3}\right)$ by Lemma 8.1 in the appendix, it suffices to check that $\varphi\left(p_{1}\left(B_{3}^{\prime}\right)\right)=p_{1}\left(B_{3}\right)$.

If $H^{*}\left(B_{3}^{\prime} ; \mathbb{Q}\right) \cong H^{*}\left(B_{3} ; \mathbb{Q}\right)$ is isomorphic to $H^{*}\left(\left(\mathbb{C} P^{1}\right)^{3} ; \mathbb{Q}\right)$, then $p_{1}\left(B_{3}^{\prime}\right)=$ $p_{1}\left(B_{3}\right)=0$ by Lemma 7.3, in particular, $\varphi\left(p_{1}\left(B_{3}^{\prime}\right)\right)=p_{1}\left(B_{3}\right)$. Suppose that $H^{*}\left(B_{3}^{\prime} ; \mathbb{Q}\right) \cong H^{*}\left(B_{3} ; \mathbb{Q}\right)$ is not isomorphic to $H^{*}\left(\left(\mathbb{C} P^{1}\right)^{3} ; \mathbb{Q}\right)$. Then Lemma 7.2 says that there is no element $\sum_{i=1}^{3} a_{i} y_{i}$ for rational numbers $a_{i}$ with $a_{3} \neq 0$ such that $\left(\sum_{i=1}^{3} a_{i} y_{i}\right)^{2}=0$. On the other hand, $y_{1}^{2}=\left(a / 2 y_{1}+y_{2}\right)^{2}=0$ and $y_{1}$ and $a / 2 y_{1}+y_{2}$ generate the subring $H^{*}\left(B_{2} ; \mathbb{Q}\right)$. The same holds for $B_{3}^{\prime}$. It follows that the images of $y_{1}^{\prime}$ and $a^{\prime} / 2 y_{1}^{\prime}+y_{2}^{\prime}$ by $\varphi$ generate the subring $H^{*}\left(B_{2} ; \mathbb{Q}\right)$, and hence $\varphi\left(H^{*}\left(B_{2}^{\prime}\right)\right) \subset H^{*}\left(B_{2}\right)$. Therefore Proposition 2.2 can be applied and we conclude that $\varphi\left(p_{1}\left(B_{3}^{\prime}\right)\right)=p_{1}\left(B_{3}\right)$ because $p\left(B_{2}^{\prime}\right)=p\left(B_{2}\right)=1$.

\section{Appendix}

In this appendix, we prove a general fact used in the previous section on StiefelWhitney classes. In the following, cohomology will be taken with $\mathbb{Z} / 2$ coefficients unless otherwise stated. Let $M$ be a connected closed manifold of dimension $n$ and let

$$
\mathrm{Sq}(x)=x+\mathrm{Sq}^{1}(x)+\mathrm{Sq}^{2}(x)+\cdots+\mathrm{Sq}^{n}(x) \quad \text { for } x \in H^{*}(M)
$$

denote the total squaring operation, where $\mathrm{Sq}^{k}: H^{q}(M) \rightarrow H^{q+k}(M)$ is an additive homomorphism. The $k$-th Wu class $v_{k}(M) \in H^{k}(M)$ of $M$ is characterized by

$$
v_{k}(M) \cup x=\operatorname{Sq}^{k}(x) \text { for any } x \in H^{n-k}(M)
$$


and

$$
\operatorname{Sq}(v(M))=w(M),
$$

where $v(M)=1+v_{1}(M)+v_{2}(M)+\cdots+v_{n}(M)$ and $w(M)$ denotes the total Stiefel-Whitney class of $M$; see [10, p.132].

Lemma 8.1. Suppose that $H^{*}(M)$ is generated by $H^{r}(M)$ for some $r$ as a ring and let $M^{\prime}$ be another connected closed manifold of dimension $n$ such that $H^{*}\left(M^{\prime}\right)$ is isomorphic to $H^{*}(M)$ as a ring. Then $\phi\left(w\left(M^{\prime}\right)\right)=w(M)$ for any ring isomorphism $\phi: H^{*}\left(M^{\prime}\right) \rightarrow H^{*}(M)$.

Proof. We first show that $\phi$ commutes with Sq. Since $H^{*}\left(M^{\prime}\right) \cong H^{*}(M)$ are generated by elements of degree $r, \mathrm{Sq}(y)=y+y^{2}$ and $\mathrm{Sq}(\phi(y))=\phi(y)+\phi(y)^{2}$ for $y \in H^{r}\left(M^{\prime}\right)$; so $\phi(\operatorname{Sq}(y))=\operatorname{Sq}(\phi(y))$. This implies that $\phi(\operatorname{Sq}(y))=\operatorname{Sq}(\phi(y))$ for any $y \in H^{*}\left(M^{\prime}\right)$ because both $\phi$ and Sq are ring homomorphisms and $H^{*}\left(M^{\prime}\right) \cong$ $H^{*}(M)$ are generated by elements of degree $r$ as rings.

It follows from (8.1) and the commutativity of $\phi$ and Sq that

$$
\phi\left(v_{k}\left(M^{\prime}\right)\right) \cup \phi(y)=\phi\left(\mathrm{Sq}^{k}(y)\right)=\mathrm{Sq}^{k}(\phi(y))
$$

for any $y \in H^{n-k}\left(M^{\prime}\right)$. Since $\phi\left(H^{n-k}\left(M^{\prime}\right)\right)=H^{n-k}(M)$, the above identity together with (8.1) implies

$$
\phi\left(v_{k}\left(M^{\prime}\right)\right)=v_{k}(M) .
$$

It follows from (8.2), (8.3) and the commutativity of $\phi$ and Sq that

$$
\phi\left(w\left(M^{\prime}\right)\right)=\phi\left(\operatorname{Sq}\left(v\left(M^{\prime}\right)\right)\right)=\operatorname{Sq}\left(\phi\left(v\left(M^{\prime}\right)\right)\right)=\operatorname{Sq}(v(M))=w(M),
$$

proving the lemma.

\section{REFERENCES}

[1] A. Borel and F. Hirzebruch, Characteristic classes and homogeneous spaces I, Amer. J. Math., 80: 458-538, 1958. MR0102800 (21:1586)

[2] S. Choi, T. Panov and D. Y. Suh, Toric cohomological rigidity of simple convex polytopes, arXiv:0807.4800.

[3] S. Choi, M. Masuda and D. Y. Suh, Quasitoric manifolds over a product of simplices, Osaka J. Math. (to appear), arXiv:0803.2749.

[4] M. Grossberg and Y. Karshon, Bott towers, complete integrability, and the extended character of representations, Duke Math. J., 76: 23-58, 1994. MR1301185 (96i:22030)

[5] P. E. Jupp, Classification of certain 6-manifolds, Proc. Cambridge Philos. Soc., 73: 293-300, 1973. MR0314074 (47:2626)

[6] P. Kleinschmidt, A classification of toric varieties with few generators, Aequationes Math. 35: 254-266, 1988. MR954243 (89f:14056)

[7] M. Masuda, Equivariant cohomology distinguishes toric manifolds, Adv. Math. 218: 20052012, 2008. MR2431667

[8] M. Masuda and T. E. Panov, Semifree circle actions, Bott towers, and quasitoric manifolds, Sbornik Math. 199:8, 1201-1223, 2008. MR2452268

[9] M. Masuda and D. Y. Suh, Classification problems of toric manifolds via topology, Proc. of Toric Topology, Contemp. Math. 460: 273-286, 2008, arXiv:0709.4579.

[10] J. W. Milnor and J. D. Stasheff, Characteristic Classes, Ann. of Math. Studies 76, Princeton N.J. 1974. MR0440554 (55:13428) 
[11] F. P. Peterson, Some remarks on Chern classes, Ann. of Math. (2), 69: 414-420, 1959. MR0102807 (21:1593)

[12] C. T. C. Wall, Classification problems in differential topology. V. On certain 6-manifolds Invent. Math., 1: 355-374, 1966. MR0215313(35:6154)

Department of Mathematical Sciences, Kaist, 335 Gwahangno, Yuseong-gu, Daejeon 305-701, Republic of Korea

E-mail address: choisy@kaist.ac.kr

Department of Mathematics, Osaka City University, Sugimoto, Sumiyoshi-Ku, Osaka 558-8585, JAPAN

E-mail address: masuda@sci.osaka-cu.ac.jp

Department of Mathematical Sciences, KAIST, 335 Gwahangno, Yuseong-gu, Daejeon 305-701, RePublic OF KoreA

E-mail address: dysuh@math.kaist.ac.kr 